\title{
Aspectos demográficos de una muestra de pacientes colombianos con diabetes mellitus tipo 1
}

\author{
Diana Clobeth Sarrazola Yepes ${ }^{1}$, Natalia Gómez-Lopera ${ }^{1}$, Jorge García ${ }^{2}$, María Victoria Lopera ${ }^{3}$, \\ Martín Toro ${ }^{3}$, Alejandra Vélez $z^{4}$ Juan Manuel Alfaro ${ }^{1,5}$ \& Nicolás Pineda-Trujillo ${ }^{1^{*}}$
}

\author{
${ }^{1}$ Grupo Mapeo Genético, Departamento de Pediatría, Facultad de \\ Medicina, Universidad de Antioquia. Medellín, Colombia. \\ ${ }^{2}$ Instituto Antioqueño de Diabetes, Medellín, Colombia. \\ ${ }^{3}$ IPS Universitaria, Universidad de Antioquia, Medellín, Colombia. \\ ${ }^{4}$ Pontificia Universidad Bolivariana, Medellín, Colombia. \\ ${ }^{5}$ Sección de Endocrinología Pediátrica, Departamento de Pediatría, \\ Facultad de Medicina, Universidad de Antioquia. Medellín, \\ Colombia.
}

Correspondencia: Nicolás Pineda-Trujillo MSc, PhD, Grupo Mapeo Genético, Departamento de Pediatría, Facultad de Medicina, Universidad de Antioquia. Carrera 51D \# 62-29,

Tel. (4) 2196065.

Correo electrónico: nicolas.pineda@udea.edu.co

Fecha de recepción: 27/07/2017

Fecha de aceptación: 18/10/2017

\section{Resumen}

$\mathrm{L}$ a diabetes mellitus tipo 1 (DM1) es una enfermedad autoinmune crónica de aparición en la infancia y el adulto joven. El subtipo autoinmune (DM1A) es más frecuente en personas con ancestría europea, mientras que el subtipo idiopático (DM1B) es más común en poblaciones de ancestría africana y asiática. Acá presentamos las características demográficas de un grupo de familias "paisas" con al menos un hijo con DM1. Se incluyó una muestra de 200 familias nucleares a las cuales se les aplicó una encuesta que preguntó aspectos generales como género, edad de diagnóstico, origen de los padres, abuelos y bisabuelos. Además se obtuvo la información sobre los autoanticuerpos anti-INS, anti-GAD, anti-ICAs y anti-TPO en 100 de los niños con diagnóstico de DM1. Se encontró que la edad media de diagnóstico fue 7,4 años. El 55\% de las familias provenían del oriente antioqueño y el Valle de Aburrá. El 8,4\% de los niños tenían un hermano con DM1. Además se pudo determinar que el $80,1 \%$ de los niños padecen DM1A, pues estos presentaban al menos un autoanticuerpo. Los resultados sobre autoinmunidad y la edad de diagnóstico permiten asimilar esta muestra con poblaciones europeas, en vez de africanos o asiáticos.

Palabras clave: Diabetes tipo 1, Antioquia, Genética, anticuerpos, demografía.

\section{Abstract}

Type 1 diabetes (T1D) is a chronic autoimmune disease with onset at infancy and early adulthood. The autoimmune subtype (T1DA) is the most frequent in people with European ancestry, whilst the idiopathic subtype (T1DB) is the commonest in populations of African or Asian ancestry. Here we present the demographic features of a set of "paisa" families which had al least one child with T1D. Two hundred nuclear families were included. An instrument asking for general information such as gender, age at diagnosis, origin of their parents, grandparents and greatgrandparents was applied. In addition, auto-antibodies data for INS, GAD, IA2 and TPO were obtained in a sub-set of 100 patients. The age at diagnosis was 7,4 years. 55\% of the families came from "Oriente", "Norte" and "Suroeste" sub-regions. 8,4\% of the children had one sib with the disease. Besides, it could be determined that $80,1 \%$ of the tested sample had T1DA, since they presented at least one type 1 diabetes related auto-antibody. Our results on autoimmunity and age at diagnosis let us assimilate this sample with European populations, rather than Africans or Asians.

Key words: type 1 diabetes, Antioquia, genetics, antibodies, demography.

\section{Introducción}

La diabetes mellitus tipo 1 (DM1) es una enfermedad metabólica de aparición temprana, siendo considerada la enfermedad crónica más común en niños y en adultos jóvenes. Se caracteriza por una total disminución en la producción de la insulina, como resultado de la destrucción selectiva de las células $\beta$ pancreáticas $y$, por consiguiente, al aumento en los niveles sanguíneos de glucosa ${ }^{(1)}$. La DM1 se ha dividido en dos sub-categorías. El subtipo A o autoinmune (DM1A) y el subtipo B o idiopático (DM1B). La DM1A es la forma más común de DM1 en la población europea. Este tipo de diabetes representa cerca del 90\% de los casos a nivel mundial; se caracteriza por la presencia de autoanticuerpos contra las células $\beta$ del páncreas como anti-GAD, anti-ICAs, anti-IA2, anti-INS. DM1B incluye los casos restantes de DM1, en esta forma de la enfermedad no se observa evidencia de una destrucción autoinmune de las células $\beta$ pancreáticas; y es más común en poblaciones con ancestría africana o asiática ${ }^{(1,2)}$. 
DM1 es una enfermedad de origen multifactorial, encontrándose asociada a factores tanto ambientales como genéticos. La influencia de un factor ambiental se evidencia por estudios en gemelos que demuestran que si un gemelo monocigoto padece DM1, el otro tendrá un riesgo cerca del $40 \%$ de padecer también esta enfermedad; es decir, la concordancia no es del $100 \%$. Sin embargo, para gemelos dicigotos esta concordancia es tan solo del $10 \%$. Este último valor es muy similar al hallado en hermanos no gemelares, lo que deja de manifiesto un factor genético influenciando el riesgo de padecer DM1 ${ }^{(3,4)}$.

Entre los factores ambientales asociados se encuentra la infección por algunos virus como el coxsackie ${ }^{(5,6)}$, citomegalovirus $^{(7)}$ y rotavirus ${ }^{(8)}$. Además se ha establecido asociación con algunos alimentos, principalmente con el consumo de leche bovina en los primeros meses de vida ${ }^{(9)}$.

Por otro lado, se ha demostrado la asociación de varios genes con el riesgo de padecer DM1. Hasta la fecha se han descrito más de 50 loci que contribuyen al riesgo genético de padecer esta enfermedad. Los genes más fuertemente asociados con el riesgo genético para DM1 han sido la región HLA y los genes PTPN22, INS y el ILRA2, entre otros [revisado en ${ }^{(10,11)}$ ]. Para Colombia, y más específicamente para Antioquia, recientemente hemos encontrado que variantes en los genes PTPN22 y IFIH1 juegan un papel en la susceptibilidad a DM1 ${ }^{(11)}$. Además, también se han implicado variantes de riesgo/protección para Antioquia en los genes INS, SUMO4, IL2RA y CLEC16 [Rodríguez et al. En preparación]. Es interesante que como parte de estos estudios excluimos al gen CTLA4 ${ }^{(11)}$.

La incidencia de DM1 varía alrededor del mundo, siendo tan s0lo de 0,1 por 100.000 menores de 14 años por año en China y Venezuela, y alcanzando valores hasta de 37 por 100.000 menores de 14 años por año en países europeos como Italia (Cerdeña) y Finlandia ${ }^{(9,12)}$.

Existen diferencias en la prevalencia y presentación de la DM1 alrededor del mundo según los diferentes grupos étnicos. Similar a Finlandia, los demás países escandinavos tienen altas tasas de incidencia. Además, países de ancestría europea como Canadá, Estados Unidos y Nueva Zelanda tienen también altas tasas de incidencia de DM1. En Latinoamérica, al igual que en África se reportan incidencias moderadas a bajas de la enfermedad. En general, en los países asiáticos se reportan las menores tasas de incidencia, siendo Kuwait la excepción, al presentar una incidencia de 22,3 por 100.000 menores de 14 años por año ${ }^{(12,13)}$.

Para la década de los 90 se estimó un aumento en la incidencia de la enfermedad del 5,3\% en Suramérica, la cual puede estar relacionada con un mayor acceso al sistema de salud y/o con factores ambientales ${ }^{(14)}$. Para esta misma década, en Colombia se estimó una incidencia de 3,8 por 100.000 menores de 14 años por año y una prevalencia de 1,8\% para la ciudad de Bogotá ${ }^{(15)}$. En otro estudio realizado en la ciudad de Medellín (Antioquia), entre los años 2001-2003, se encontró que el 4\% de los pacientes diagnosticados con diabetes mellitus en esta ciudad, padecen DM1 $1^{(16)}$.
La población colombiana pertenece al grupo étnico denominado mixto, conformado por la mezcla entre personas caucásicas, afrodescendientes y amerindios, cuyas proporciones varían a través de las diferentes regiones del país ${ }^{(17)}$. El departamento de Antioquia está ubicado al noroeste de Colombia, en la región Andina y cuenta con un área de aproximadamente $63.000 \mathrm{Km}^{2}$. Se encuentra dividido en 125 municipios, los cuales se agrupan en 9 subregiones denominadas Nordeste, Occidente, Valle de Aburrá, Oriente, Suroeste, Bajo Cauca, Norte, Magdalena Medio y Urabá. La región "paisa" comprende un área cultural y geográfica delimitada. Ésta se encuentra conformada por los departamentos de Antioquia (excluyendo la subregión del Urabá), Risaralda, Quindío y Caldas, y los municipios ubicados en el norte de los departamentos de Tolima y del Valle del Cauca.

En el presente estudio se analizan los aspectos demográficos de una muestra de 207 niños "paisas" diagnosticados con DM1 y sus familias, así como su analogía con los diferentes grupos étnicos de los cuales se deriva esta población.

\section{Metodología}

\section{Muestra}

Para el análisis se contó con una base de datos de 200 familias "paisas" que tuvieran al menos un hijo con DM1 (caso índice). Las familias fueron captadas por el caso índice en el servicio de endocrinología del Hospital Universitario San Vicente Fundación, la IPS Universitaria de la Universidad de Antioquia, el Instituto Antioqueño de Diabetes y la Universidad Pontificia Bolivariana, de Medellín. Para la elección de las familias se tuvieron los siguientes criterios de inclusión: (a) Ancestría "paisa" auto-reportada, definida como la presencia de al menos seis de los bisabuelos nacidos en territorio "paisa" (figura 1). (b) Tener diagnóstico clínico y por el laboratorio de DM1, con base en los criterios establecidos por la Asociación Americana de Diabetes ${ }^{(1)}$. (c) Contar con la participación de ambos padres.

A cada una de estas familias se les aplicó una encuesta de ancestralidad, la cual incluyó aspectos generales como la edad de diagnóstico, el género, el origen de los padres, abuelos y bisabuelos y la historia familiar de DM1 u otras enfermedades autoinmunes.

\section{Evaluación de auto-anticuerpos}

Se obtuvieron los datos de los principales auto-anticuerpos (AACs) asociados con el desencadenamiento de DM1, tales como anti-GAD, anti-IA2 y anti-INS. Estos tres AACs se evaluaron usando un kit basado en inmunofluorescencia [Kronus, Boise, ID, USA $]^{(18)}$. También se incluyeron los anticuerpos contra la tiro-peroxidasa (anti-TPO), los cuales fueron evaluados usando un kit basado radio-inmunoensayo 6 [Kronus, Boise, ID, USA] ${ }^{(18)}$.

\section{Análisis estadístico}

La información se colectó y procesó en una base de datos en Microsoft Excel 2007. En este mismo software se calcularon los 
Figura 1. Región "paisa" en Colombia. Esta la componen parte del departamento de Antioquia, los departamentos de Caldas, Risaralda y Quindío. Además, también hacen parte el norte de los departamentos del Valle del Cauca y del Tolima. Figura tomada de http://www.fotoseimagenes.net/region-paisa

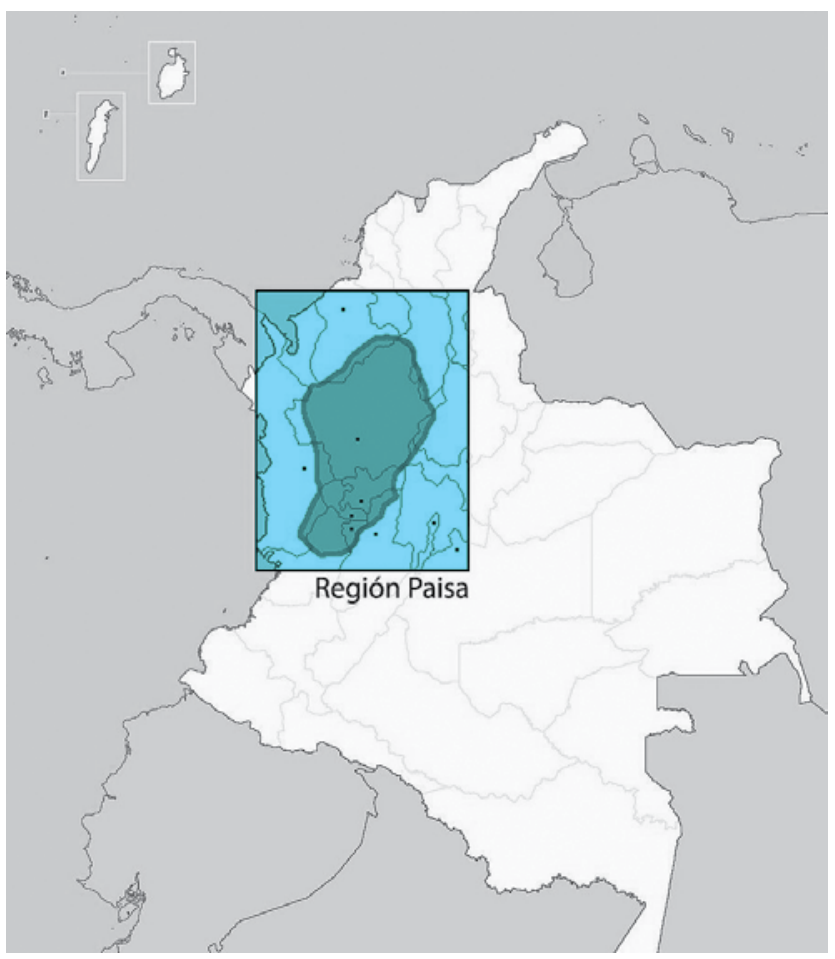

porcentajes para las diferentes categorías. Los porcentajes de casos según su género se evaluaron usando la chi-cuadrada. Las figuras fueron obtenidas usando el paquete CRAN-R ${ }^{(19)}$.

\section{Resultados}

Se obtuvieron 200 familias nucleares antioqueñas, de las cuales 193 correspondían a tríos conformados por un hijo afectado, el padre y la madre, y siete familias conformadas por dos hijos afectados, el padre y la madre. Así, en total contamos con 207 niños con DM1, de los cuales el 57,5\% (115) fueron hombres y el 42,5\% (92) fueron mujeres, sin evidencia de diferencias estadísticamente significativas entre ambos $(p=0,11)$. Su edad promedio de diagnóstico fue 7,4 $\pm 4,7$ años (figura 1).

Con relación a la historia familiar de DM1, encontramos que de los 207 niños, el 8,7\% (18) tienen al menos un hermano con DM1. El 2,9\% (6) de los padres de los niños también padecen DM1 y el 20,8\% (43) de los niños tienen un familiar cercano con DM1. Además se encontró que el 19,3\% (40) de los niños tienen algún familiar con otra enfermedad autoinmune, principalmente enfermedad tiroidea y artritis (figura 2).

$\mathrm{Al}$ evaluar las sub-regiones de las cuales provenían los bisabuelos de los niños afectados, se encontró que la mayoría de ellos provienen del oriente antioqueño y el Valle de Aburrá, con
Figura 2. Edad de diagnóstico e historia familiar de DM1

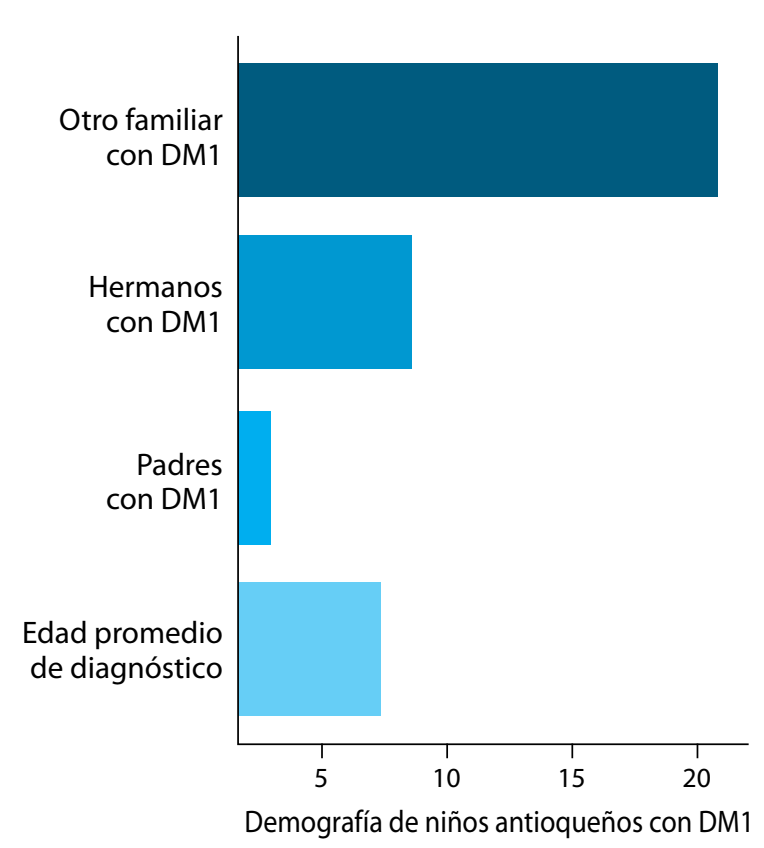

una frecuencia de $23 \%$ cada una. El 17\% provenía del suroeste, y el 15\% del norte de Antioquia. Frecuencias más bajas para la procedencia de los bisabuelos se reportan en el occidente (7\%), el nordeste (4\%) y de otras regiones paisas no pertenecientes al departamento de Antioquia (5\%) (figura 3).

Con base en la medición de autoanticuerpos en los 105 niños se encontró que el 80,1\% de ellos padecen DM1 autoinmune y solo el 19\% padecen de DM1B. Se encontró que el autoanticuerpo más común es el anti-INS pero dado que todos los niños fueron captados cuando ya habían iniciado terapia con insulina exógena, este dato no se tuvo en cuenta para el análisis. Con relación a los otros tres autoanticuerpos, el más común fue el anti-IA2 con un 69,5\%, seguido por el anti-GAD con un $42,9 \%$ y en menor proporción el anti-TPO con un 11,36\% (tabla 1).

\section{Discusión}

El presente estudio define las características demográficas de un grupo de pacientes colombianos con DM1. En Colombia existen pocos reportes de estudios donde se indaguen la prevalencia, incidencia y características generales de la DM1. Con base en un estudio realizado en la década de 1990, en la ciudad de Bogotá (Colombia) ${ }^{(18)}$, en el cual se reporta una incidencia de DM1 del 3,8 por 100.000 habitantes menores de 14 años, Barceló y Rajpathak en el $2001^{(20)}$ calcularon una prevalencia de 1,8 por 10.000 habitantes para esta región. Sin embargo, no se cuenta con datos de incidencia o prevalencia de DM1 para la región "paisa".

En la población analizada se encontró una mayor proporción de pacientes masculinos $(57,5 \%)$ en relación con los de sexo femenino (42,5\%). Proporciones similares son reportadas 


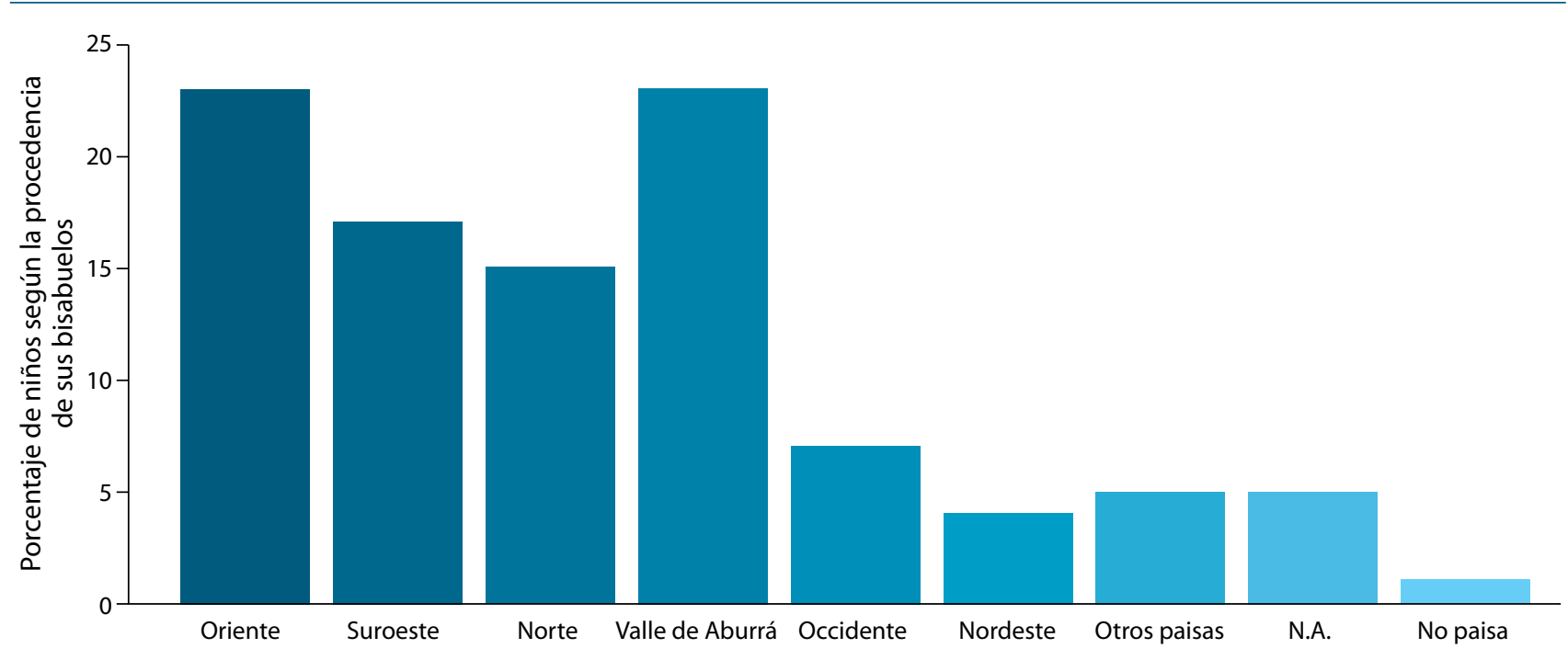

Tabla 1. Porcentaje de anticuerpos en 105 niños con DM1

\begin{tabular}{l|c|c|c|c|c} 
& Anti-GAD & Anti-IA2 & Anti-INS & Anti-TPO & Autoinmunidad* \\
\hline Positivo & $42,90 \%$ & $71,57 \%$ & $98,20 \%$ & $11,36 \%$ & $80,10 \%$ \\
\hline Negativo & $57,10 \%$ & $28,43 \%$ & $1,80 \%$ & $88,64 \%$ & $19,00 \%$ \\
\hline
\end{tabular}

*Excluyendo los datos de Anti-INS

en países con altas incidencias de DM1, principalmente en poblaciones descendientes de europeos como la austriaca ${ }^{(15)}$. Por el contrario, en países con incidencias más bajas se halla una mayor proporción de población femenina afectada, con relación a la masculina ${ }^{(21)}$.

El porcentaje de hermanos afectados $(8,7 \%)$ hallado en este estudio fue similar a los valores reportados en la literatura para hermanos no gemelares, para quienes se han hallado valores de concordancia del 6,5\% y 7\% para población finlandesa y danesa, respectivamente ${ }^{(14)}$.

En un estudio realizado en Estados Unidos donde se reporta la incidencia para los diferentes grupos étnicos según la edad de diagnóstico, se encontró que la mayoría de pacientes eran diagnosticados en la adolescencia, entre los 10 y 14 años, seguido por el grupo entre 5 y 10 años ${ }^{(22,23)}$. En nuestro estudio se encontró que la edad promedio de diagnóstico fue de 7,4 años y solo 14 pacientes tenían una edad superior a los 14 años al momento del diagnóstico. Los promedios en la edad de diagnóstico reportados acá concuerdan con la literatura para poblaciones europeas y suramericanas ${ }^{(24)}$.

En la población estudiada se encontró que el 80,1\% de los pacientes tenían al menos un autoanticuerpo contra las células $\beta$ del páncreas. Este valor es menor al reportado previamente en otro estudio usando pacientes provenientes de la misma población ${ }^{(25)}$. Sin embargo, en ese estudio se tuvieron en cuenta los valores de autoanticuerpos contra la insulina, datos que fueron obviados en nuestro análisis, dado que los pacientes estaban recibiendo tratamiento con insulina exógena, lo cual podría llevar a confusión en los resultados.

Cuando se toman solo los valores de anti-GAD se encuentra que el $42,9 \%$ de los pacientes son positivos mientras que para anti-IA2 se encontró positividad en el $71,57 \%$ de estos. Datos que contrastan con lo reportado para el Reino Unido, donde se halla que el $79,4 \%$ y $78 \%$ son positivos para anti-GAD y anti-IA2 respectivamente ${ }^{(26)}$. No obstante, nuestros resultados son comparables con los obtenidos en un estudio realizado en España en la provincia de Palencia, donde se encontró que el 50\% de los pacientes diagnosticados con DM1 eran positivos para anti-GAD y el $73,1 \%$ para anti-IA2 ${ }^{(27)}$. Los valores hallados para anti-TPO no necesariamente se correlacionan con la presencia de alguna afección de la tiroides, pues esta función no fue evaluada en este estudio. A pesar de que los valores encontrados por nosotros $(11,36 \%)$ son similares a los encontrados en un estudio realizado en el Reino Unido, donde encontraron que $10,8 \%$ de los casos eran positivos para este autoanticuerpo ${ }^{(28)}$. Es interesante que en ese estudio se reportó un sesgo de casos de género femenino con la enfermedad, mientras que nuestro estudio muestra una mayor frecuencia (no significativa) hacia el género masculino.

La mayoría de los pacientes incluidos provienen de las subregiones oriente, norte y suroeste, las cuales comparten un 
tronco de poblamiento del territorio en la época de la colonización. Los pacientes de estas tres subregiones comprenden el $55 \%$ de la población analizada, lo cual sugiere homogeneidad genética en sus variantes de susceptibilidad. Siendo la DM1 una enfermedad con prevalencias más altas en poblaciones de origen europeo, este dato se correlaciona con los antecedentes históricos de la región.

Antioquia fue fundada a partir de dos núcleos principales, Marinilla, en la subregión oriente, y Santa Fe de Antioquia, en la subregión occidente. De este último se derivó la población del Valle de Aburrá. Después de la época colonial, dadas las características geográficas de Antioquia, su población permaneció aislada del resto del país, llevando a una expansión más interna que externa, lo cual ha sido corroborado con estudios genéti$\cos ^{(29)}$, que a su vez han demostrado que esta población posee una ancestría predominantemente europea. Estos hechos se reflejan en la aparición de efectos fundadores para enfermedades con patrón de herencia recesiva ${ }^{(30)}$ o dominante ${ }^{(31)}$.
En conclusión, en la muestra de pacientes con DM1 presentados acá, se esperaría encontrar variantes genéticas similares a las reportadas en estudios realizados en poblaciones de origen europeo. No obstante, no se descarta el fenómeno de nuevas variantes asociadas al riesgo/protección. Es decir, que variantes no reportadas anteriormente en los genes ya implicados, resulten asociadas en nuestra población, como fue el caso para la variante rs10930046 en el gen $I F I H 1^{(11)}$. Este hallazgo refuerza la importancia de diseñar los estudios de réplica, pensando en la contribución que el gen puede hacer a la susceptibilidad/protección, más que la mera variante ya reportada en otra población.

\section{Agradecimientos}

Estamos muy agradecidos con los pacientes que participaron en este estudio; también agradecemos a sus padres por su vinculación al estudio.

Agradecemos el apoyo financiero de la Universidad de Antioquia y del CODI-UdeA (grant \# 2548).

\section{Referencias}

1. ADA. Classification and Diagnosis of Diabetes. Diabetes Care. 2016 Jan;39(Supplement 1):S13-22.

2. Noble J a, Erlich $\mathrm{H}$ a. Genetics of type 1 diabetes. Cold Spring Harb Perspect Med. 2012 Jan;2(1):a007732.

3. Condon J, Shaw JE, Luciano M, Kyvik KO, Martin NG, Duffy DL. A Study of Diabetes Mellitus Within a Large Sample of Australian Twins. Twin Res Hum Genet. 2008 Feb 21;11(1):28-40.

4. Nisticò L, Iafusco D, Galderisi A, Fagnani C, Cotichini R, Toccaceli V, et al. Emerging Effects of Early Environmental Factors over Genetic Background for Type 1 Diabetes Susceptibility: Evidence from a Nationwide Italian Twin Study. J Clin Endocrinol Metab. 2012 Aug;97(8):E1483-91.

5. Yeung W-CG, Rawlinson WD, Craig ME. Enterovirus infection and type 1 diabetes mellitus: systematic review and meta-analysis of observational molecular studies. BMJ. 2011 Jan;342:d35.

6. Hober D, Sane F. Enteroviruses and type 1 diabetes. BMJ. 2011 Jan;342:c7072.

7. Aarnisalo J, Veijola R, Vainionpää R, Simell O, Knip M, Ilonen J. Cytomegalovirus infection in early infancy: risk of induction and progression of autoimmunity associated with type 1 diabetes. Diabetologia. 2008 May;51(5):769-72.

8. Ballotti S, de Martino M. Rotavirus Infections and Development of Type 1 Diabetes: An Evasive Conundrum. J Pediatr Gastroenterol Nutr. 2007 Aug; 45(2):147-56.

9. Egro FM. Why is type 1 diabetes increasing? J Mol Endocrinol. 2013 Jan;51(1):R1-13.

10. Barrett JC, Clayton DG, Concannon P, Akolkar B, Cooper JD, Erlich H a, et al. Genome-wide association study and meta-analysis find that over 40 loci affect risk of type 1 diabetes. Nat Genet. 2009 May;41(6):703-7.

11. Rodríguez A, Alfaro JM, Balthazar V, Pineda Trujillo N. Association analysis of PTPN22, CTLA4 and IFIH1 genes with type 1 diabetes in Colombian families. J Diabetes. 2015;7:402-10.

12. Patterson CC, Gyürüs E, Rosenbauer J, Cinek O, Neu a, Schober E, et al. Trends in childhood type 1 diabetes incidence in Europe during 1989-2008: evidence of non-uniformity over time in rates of increase. Diabetologia. 2012 Aug;55(8):2142-7.

13. Ludvigsson J. Why diabetes incidence increases--a unifying theory. Ann N Y Acad Sci. 2006 Oct;1079:374-82.

14. The Diamond Project Group. Incidence and trends of childhood Type 1 diabetes worldwide 1990-1999. Diabet Med. 2006 Aug;23(8):857-66.

15. Barceló A, Rajpathak Ss. Incidence and prevalence of diabetes mellitus in the Americas. Rev Panam salud pública/Pan Am J Public Heal. 2001 Nov;10(5):300-8.

16. Villegas Perrase A, Abad SB, Faciolince S, Maya C, Parra L, Rivas E. El control de la diabetes mellitus y sus complicaciones en Medellín, Colombia, 20012003. Rev Panam salud pública/Pan Am J Public Heal. 2006;20(6):393-402.

17. Bedoya G, Montoya P, García J, Soto I, Bourgeois S, Carvajal L, et al. Admixture dynamics in Hispanics: a shift in the nuclear genetic ancestry of a South American population isolate. Proc Natl Acad Sci U S A. 2006 May 9;103(19):7234-9.
18. Gutiérrez-achury J, Balthazar-gonzález V, Bedoya-berrío G, Ruíz-linares A, Uribe-londoño F, Alfaro JM, et al. Association of the TPO gene in Colombian families with type 1 diabetes. Iatreia. 2009;22(4):323-9.

19. R Core Team. R: A language and environment for statistical computing. Vienna, Austria; 2014.

20. Karvonen M, Viik-Kajander M, Libman I, LaPorte R, Tuomilehto J. Incidence of Childhood Type 1 Diabetes Worldwide. Diabetes Care. 2000;23(10):151626.

21. Catanzariti L, Faulks K, Moon L, Waters a-M, Flack J, Craig ME. Australia's national trends in the incidence of Type 1 diabetes in 0-14-year-olds, 20002006. Diabet Med. 2009 Jun;26(6):596-601.

22. Harjutsalo V, Podar T, Tuomilehto J. Cumulative Incidence of Type 1 Diabetes in 10,168 Siblings of Finnish Young-Onset Type 1 Diabetic Patients. Diabetes. 2005;54(2):563-9.

23. Kyvik KO, Green a, Beck-Nielsen H. Concordance rates of insulin dependent diabetes mellitus: a population based study of young Danish twins. BMJ. 1995 Oct;311(7010):913-7.

24. Dabelea D, Bell R a, D'Agostino RB, Imperatore G, Johansen JM, Linder B, et al. Incidence of diabetes in youth in the United States. JAMA. 2007 Jun;297(24):2716-24.

25. Tobon GJ, Arango A, Abad V, García J, Cuervo H, Velásquez A, et al. Clinical and immunological characteristics of type 1 diabetes mellitus in a northwestern Colombian population. Diabetes Res Clin Pract. 2006 May;72(2):170-5.

26. Long AE, Gillespie KM, Rokni S, Bingley PJ, Williams AJK. Rising Incidence of Type 1 Diabetes Is Associated With Altered Immunophenotype at Diagnosis. Diabetes. 2012 Mar 1;61(3):683-6.

27. Bertholt ML, Maldonado Ruiz E, Gonzalez De la Torre M de la C, Rubiera Perez G, De Llano JA. Características de la diabetes mellitus tipo 1 al debut . Evolución de la patología durante los últimos 21 años en un hospital de referencia de segundo nivel . Rev Española Endocrinol Pediatr. 2012;3(1):52-7.

28. Howson JMM, Dunger DB, Nutland S, Stevens H, Linda S, Todd JA. A type 1 diabetes subgroup with a female bias is characterised by failure in tolerance to thyroid peroxidase at an early age and a strong CTLA-4 gene association. Diabetologia. 2007;50(4):741-6.

29. Carvajal-Carmona LG, Soto ID, Pineda N, Ortiz-Barrientos D, Duque C, OspinaDuque J, et al. Strong Amerind/White Sex Bias and a Possible Sephardic Contribution among the Founders of a Population in Northwest Colombia. Am J Hum Genet. 2000 Nov;67(5):1287-95.

30. Pineda-Trujillo N, Apergi M, Moreno S, Arias W, Lesage S, Franco A, et al. A genetic cluster of early onset Parkinson's disease in a Colombian population. Am J Med Genet B Neuropsychiatr Genet. 2006 Dec;141B(8):885-9.

31. Lopera F, Ardilla A, Martínez A, Madrigal L, Arango-Viana JC, Lemere CA, et al. Clinical features of early-onset Alzheimer disease in a large kindred with an E280A presenilin-1 mutation. JAMA. 1997 Mar;277(10):793-9. 